\title{
Inhalt, Vol. 16, No. 6, 1993
}

\section{Contents}

Übersichtsarbeiten

Richtlinien für die Definition von Zielvolumina bei der Strahlentherapie des Prostatakarzinoms:

Übersicht und Perspektiven

Vijayakumar, S., Chan, T., Ray, V., Grant, T., Chiru, P.,

Ray, P., Chen, G.G.T.Y 389

Die Therapie des Morbus Hodgkin

Tesch, H., Engert, A., Lathan, B., Löffler, M., Hasenclever, D,

Pfreundschuh, M., Dühmke, E., Diehl, V. 407

Neue Möglichkeiten zur adjuvanten Therapie von kolorektalen Karzinomen einschließlich deutscher Aktivitäten

Hartung, G., Diezler, P., Hagmüller, E., Queißer, W.

Endoluminaler und intraoperativer Ultraschall

Kleinau, H., Liebeskind, U., Zaiac, M., Schlag, P.M 425

Review Articles

Guidelines for Defining Target Volumes in Radiation Therapy of Prostate Cancer: a Review and Perspective

Vijayakumar, S., Chan, T., Ray, V., Grant, T., Chiru, P.,

Ray, P., Chen, G.G.T.Y. 389

Therapy of Hodgkin's Disease

Tesch, H., Engert, A., Lathan, B., Löffler, M., Hasenclever, D.,

Pfreundschuh, M, Dühmke, E., Diehl, V. 407

New Approaches to Adjuvant Therapy of Colorectal Carcinoma Including Current German

Activities

Hartung, G, Diezler, P., Hagmüller, E., Queißer, W. 416

Endoluminal and Intraoperative Ultrasound

Kleinau, H, Liebeskind, U, Zaiac, M, Schlag, P.M 425

Originalarbeiten

Gefäßinvasion und Frührezidiv beim Mammakarzinom: Vorläufige Ergebnisse

Obermair, A., Czerwenka, K., Kurz, C, Schemper, M.,

Sevelda, P.

Mitoxantron, Etoposid und Prednisolon (NOVEP) in der Primärtherapie älterer Patienten mit

Non-Hodgkin-Lymphom und zur Therapie bei refraktärenNon-Hodgkin-Lymphomen

Haferlach, T, Gassmann, W., Uharek, L., Tiemann, M.,

Löffler, $\mathrm{H}$

Phase-II-Studie mit 4'-Iodo-4'-Deoxydoxorubicin bei Patienten mit fortgeschrittenem, meßbarem, nichtklein-zelligem Bronchialkarzinom 
Eberhardt, W, Niederle, N., Wilke, H, Weidmann, B.,

Henss, H., Engelhardt, R., Zeidler, D., Colajori, E.,

Kerpel-Fronius, S., Seeber, S

Original Paper

Vessel Invasion Predicts Early Recurrence in Breast Cancer: Preliminary Results

Obermair, A., Czerwenka, K,, Kurz, C, Schemper, M.

Sevelda, $\mathrm{P}$.

434

Mitoxantrone, Etoposide and Prednisolone (NOVEP) in Previously Untreated Elderly Patients with Non-Hodgkin's Lymphoma (NHL) and as Salvage Therapy for Refractory NHL

Haferlach, T, Gassmann, W., Uharek, L.. Tiemann, M..

440 Löffler, $\mathrm{H}$

Phase II Study of 4'-Iodo-4'-Deoxydoxorubicin in Patients with Advanced, Measurable Non-

Small Cell Lung Cancer

Eberhardt, W., Niederle, N., Wilke, H, Weidmann, B.,

Henss, H, Engelhardt, R., Zeidler, D., Colajori, E.,

446 Kerpel-Fronius, S., Seeber, $\mathrm{S}$

434

440

446

Eine Phase-I/II-Studie mit Chinidin, einer potentiell die Multidrug Resistance überwindenden Substanz, und Pirarubicin beim metastasierten, chemotherapie-refraktären Mammakarzinom Raderer, M., Depisch, D., Haider, K., Kwasny, W.,

Djavanmard, M., Scheithauer, W.

150

A Phase I/II Study of Quinidine, a Potential Multidrug Resistance-Reversing Agent, in Combination with Pirarubicin in Patients with Advanced Refractory Breast Cancer

Raderer, M., Depisch, D., Haider, K., Kwasny, W.,

Djavanmard, M, Scheithauer, W.... .

450

Fortsetzung aufSeite 388

Continued on page 388

Band 16, Heft 6, Dezember 1993

Internationale Zeitschrift für Krebsforschung und -behandlung

Inhalt

Contents

Ein venöser Mini-Port, implantiert am proximalen Unterarm, distalen Oberarm oder an der Brustwand

Berdel, W. E., Ridwelski, K,, Korfel, A., Matthias, M., Harnoss, B.-M., Boese-Landgraf, J., May, E., Thiel, E....

A Venous Access Mini-Port Implanted on the Proximal Forearm, on the Distal Upper Arm or on the Chest Wall 
Berdel, W.E., Ridwelski, K,, Korfel, A., Matthias, M.,

454 Harnoss, B.-M., Boese-Landgraf, J., May, E., Thiel, E

454

Kurzmitteilungen

Phase-II-Studie mit niedrigdosiertem IFN-gamma beim metastasierten Nierenzellkarzinom

Scheibenbogen, C, Keilholz, U., Maclachlan, D., Möhler, T.,

Brossart, P., Hunstein, W.

Radioimmunszintigraphie von Kopf- und Halstumoren

Schomburg, A., Hotze, A.L., Walther, E. K., Alberty, J.,

Bender, H., Herberhold, C, Biersack, H.J

Buchbesprechung

Short Communications

Phase II Study of Intermittent Low-Dose IFN-gamma in Metastatic Renal Cell Cancer

Scheibenbogen, C, Keilholz, U., Maclachlan, D., Möhler, T.,

461 Brossart, P., Hunstein, W.

461

Radioimmunoimaging of Head and Neck Cancer

Schomburg, A., Hotze, A.L., Walther, E. K., Alberty, J.,

465 Bender, H., Herberhold, C, Biersack, H.J 465

470 Book Review 470

Miiteilungen onkologischer Gesellschaften

Mitteilungen der Arbeitsgemeinschaft Internistische

Onkologie (AIO) 471

Mitteilungen der Berliner Krebsgesellschaft e.V. 472

Impressum 385

Inhaltsverzeichnis 386

Industrieforum 473

Autorenverzeichnis 1993

Jahres-Inhalt 1993

Sachwortregister 1993

474

479

Hinweise für Autoren 487

Reports of Oncological Societies

Reports of the Arbeitsgemeinschaft Internistische

Onkologie (AIO)

471

Reports of the Berliner Krebsgesellschaft e.V.

472

Imprint 385

Contents 386

Industrial Forum 473

Author Index 1993

Complete Contents $1993 \quad 476$

Subject Index 1993

Instructions to Authors 487

Bibliographischer Hinweis: Inhaltsverzeichnisse dieser Zeitschrift erscheinen regelmäßig in current contents ${ }^{\circledR}$ sowie in anderen bibliographischen Diensten. 